\section{Law breaking}

\author{
J. H. Mulvey
}

The New Physics. Edited by Paul Davies. Cambridge University Press: 1989. Pp. 516. $£ 30, \$ 49.50$

Claiming an omniscience sometimes irritating to other scientists, physicists regard the whole of the physical world as their domain. They dare to explain the birth of the Universe, expect to find simplicity in the midst of evident complexity, and dissect matter to find its smallest constituents and to uncover the laws ruling its behaviour. The discovery of the electron, the atomic nucleus and the laws of quantum mechanics answered the questions posed by Mendeleev's periodic table of the elements and provided the basis for our understanding of almost all modern science and technology. The first 30 years of this century, which also saw the advent of Einstein's special and general theories of relativity, are still looked upon as the golden age of physics.

Paul Davies's objective in compiling The New Physics is to advertise the advances of more recent decades, taking us on to new frontiers. To span an ambitiously broad sweep of topics he has the help of 18 contributors, all of whom are distinguished in their fields and who write in a welcome diversity of styles: flamboyant, systematic, excited, thoughtful, fanciful. The chapters are complete in themselves leading inevitably to some overlap, but this is often helpful as some authors are more successful than others in explaining unfamiliar terms and concepts. The level is generally that of Scientific American, though some contributions have tougher sections and several use mathematical language.

The first section of the book deals with physics on the scale of the Universe, where gravity reigns. The last concentrates on particle physics and the tantalizingly close approaches to a unified theory of the forces of nature. In the middle we find physics on a more human scale, dealing with systems amenable to control and manipulation in the laboratory but often showing great complexity of behaviour.

Clifford Will, Chris Isham, and Alan Guth and Paul Steinhardt review general relativity, the still elusive quantum theory of gravitation, and the inflationary version of the Big Bang model of the early Universe. The latter provides a mechanism for matter and energy to emerge out of nothing - "perhaps the ultimate free lunch". Stephen Hawking's short essay ponders the "edge of spacetime", contemplating the curvature of time as well as of space so that to ask "What came before the Big Bang?" would be like asking to go one mile north of the North Pole. In a well-illustrated 'mini-monograph' Malcolm Longair describes and interprets the vast range of observational data that has transformed our view of the Universe.

The title, "Critical Point Phenomena: Universal Physics at Large Length Scales" does not immediately evoke the flavour of fashionable 'new physics', but Alastair Bruce and David Wallace pick up one of the unifying threads running through the book. They demonstrate the significance of relationships between the lengths, or scales, characterizing a physical system and introduce the ideas underlying the

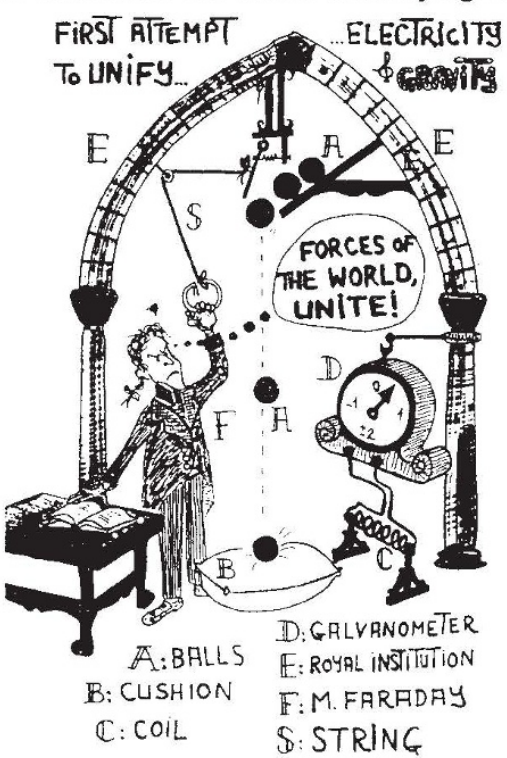

Michael Faraday at the Royal Institution, a cartoon by A. de Rujula (reproduced from The New Physics).

mathematical technique known as the renormalization group.

David Thouless describes the mobilization of armies of atoms or electrons forming linear or two-dimensional arrays in physicist-engineered materials that have many novel properties. The world of low-temperature physics, at temperatures close to absolute zero where quantum mechanics rules on a macroscopic scale, is the topic for Anthony Leggett. More examples of elegant physics are described by Peter Knight writing on quantum optics; they range from the stimulation of great orchestras of atoms to emit intense beams of laser light to the observation of a lone atom 'winking'.

The development of methods for the treatment of nonlinear processes forms a distinct branch of the new physics. Gregoire Nicolis discusses far-from-equilibrium systems and self organization, as in the regular patterns of convection in a heated liquid. Such complex systems can evolve into random, unpredictable motion and this is the subject for Joseph Ford, a self-proclaimed "disciple of chaos".

Abner Shimony, writing more in the style of a philosopher than a physicist, reviews the conceptual foundations of quantum mechanics. Frank Close explains the basis for our belief in the unobserved, perhaps unobservable quarks which are, with the electron, today's ultimate building blocks of matter. Howard Georgi makes two lively contributions. One is on Grand Unified Theories (GUTs); these attempt to find a theoretical framework unifying quantum chromodynamics (QCD), the theory describing the force between quarks, with the electroweak theory which unites electromagnetism and the weak interaction (responsible, inter alia, for radioactivity). In his second piece Georgi describes his views on the status of quantum field theories. Here he warns theoreticians against extending their speculations into energy regions too far beyond the discipline of experiment in a search for mathematical elegance.

Three of the four basic forces of nature are now understood and described by quantum field theories. They are all 'gauge forces', a term explained by John Taylor who goes on to review their quite different characters: the infinite range of electromagnetism, the short range of the weak force, and the extraordinary feature of quark confinement in QCD.

The last word is given to Abdus Salam. Fancy as ever unconstrained, he contemplates 'theories of everything' based on the concept of supersymmetric strings in as many as 26 dimensions, and speculates on the feasiblity of lunar-encircling particle acclerators. After describing some of the theoretical results, he makes a remark which can stand for the book as a whole: "One can only hope nature is aware of our work on all this".

Two impressions stand out: the remarkable unity of the new physics, and the role of curiosity as the vital driving force of discovery. Shared concepts and methods link the different sectors in ways scarcely imagined 20 years ago; examples are the inflationary model of the early Universe based on ideas from GUTs, and the significance of dimensional scales and the renormalization process which underlie approaches to problems as varied as symmetry breaking in gauge theories, critical point phenomena, self-organization, and the development of chaos. Applications are hardly, if ever, mentioned as a motive, but the 'useful products' already realized are of immense value and more will come. Here are two lessons for those, even to be found among scientists, who would manage and direct science towards 'purposeful' ends.

This is an attractively presented book, but it is one to be read and thought about, rather than merely looked at. Nonscientists will get a good sense of some of the new ideas, and scientists - physicists included - can learn how very far physics has extended its dominion

J.H. Mulvey is a Senior Research Officer in the Nuclear Physics Laboratory, University of Oxford, Keble Road, Oxford OX1 3RH, UK. 